\title{
Diagnóstico tardio de Esclerose Tuberosa
}

\author{
A delayed diagnosis of Tuberous Sclerosis
}

\author{
Catarina Oliveira ${ }^{1,2}$, Raquel Real ${ }^{2,3,}$, , Goreti Nadais ${ }^{2}$, Carolina Garrett ${ }^{2,3}$ \\ ${ }^{1}$ Department of Internal Medicine, Centro Hospitalar Tondela-Viseu, Portugal \\ ${ }^{2}$ Department of Neurology, Centro Hospitalar de São João, Portugal \\ ${ }^{3}$ Faculty of Medicine, University of Porto, Portugal \\ "Current address: MRC Clinical Sciences Centre, London, United Kingdom
}

\begin{abstract}
Tuberous sclerosis (also referred to as Tuberous Sclerosis Complex) is an autosomal dominant neurocutaneous syndrome that can involve multiple organs such as the brain, heart, kidney, lung, liver, skin and eye. The diagnosis is clinical and most patients are identified during childhood, in the context of a severe epileptic or neuropsychiatric disorder. Here we report a case of TSC diagnosed in an adult woman with dermatological and vascular involvement that were only recognized as manifestations of TSC after the diagnosis of her infant daughter. This case highlights how important it is that physicians recognize the full spectrum of manifestations of TSC, including the most unusual, so not to miss the diagnosis.
\end{abstract}

Keywords: Tuberous sclerosis complex. Facial angiofibromas. Hypomelanotic macules. Subependymal giant cell astrocytoma. Aortic aneurysm.

Palabras clave: Esclerosis tuberosa. Angiofibromas faciales complejos. Máculas hipomelanóticas. Astrocitoma subependimario de células gigantes. Aneurisma de aorta.

\section{Introduction}

Tuberous sclerosis (TC) is an autosomal dominant genetic disorder with multisystem clinical manifestations caused by mutations in either the TSC1 gene on chromosome 9 or the TSC2 gene on chromosome 16, which encode the proteins hamartin (TSC1) and tuberin (TSC2), respectively ${ }^{1,4}$. Genetic mutations in these genes lead to hyperactivation of the mammalian target of rapamycin (mTOR) pathway, which causes abnormal control of cell growth and results in tumour formation throughout the body ${ }^{2}$. Demonstration of a pathogenic mutation in TSC1 or TSC2 in normal tissue is considered sufficient for the diagnosis, independent of clinical manifestations. Despite this, clinical diagnostic criteria are important because genetic testing cannot identify the mutation in up to $25 \%$ of patients ${ }^{7}$. The expanded phenotype is known as the tuberous sclerosis complex (TSC) to emphasize the multiorgan involvement, characterized by the formation of hamartomatous lesions in the brain, heart, skin, kidney, lung, and other organs ${ }^{4,5}$. The incidence of TC is approximately 1:6000 newborns and the diagnosis typically occurs during childhood. Clinical manifestations include disabling neurological disorders, such as epilepsy, mental retardation and autism ${ }^{1,2}$. Additional major features include dermatologic lesions such as facial angiofibromas, renal angiomyolipoma (AML), retinal abnormalities, cardiac rhabdomyoma and pulmonary lymphangiomyomatosis (LAM) $)^{6,7}$ - Table 1. Renal AMLs are usually incidental findings, but they may manifest with abdominal pain and/or a palpable mass; complications include retroperitoneal haemorrhage, haematuria, hypertension or renal failure. Clinically, LAM is characterized by progressive dyspnea on exertion and recurrent pneumothorax. Most cardiac rhabdomyomas are asymptomatic, with a minority causing arrhythmia and/or cardiac failure ${ }^{7}$. Vascular manifestations of TS are now being more frequently acknowledged, and although significant vascular lesions are rare, they can be life threatening. Because of the wide range of organs affected, a multidisciplinary team approach is ideal and produces the best outcomes for patients. Treatment options for TSC have been limited, focusing primarily on the management of symptoms. However, the discovery of mTOR pathway upregulation in TSC-associated tumours presents new possibilities for treatment strategies ${ }^{1}$.

\section{Case report}

A 38 year-old woman was referred to the Neurology Clinic due to a family history of TSC - the daughter had been diagnosed with TSC at the age of 8 months in the context of epilepsy - and suspicious skin lesions. The patient denied a history of seizures or other neurological symptoms, although she reported a mild learning disability during childhood. The patient had a background of arterial hypertension and aortic arch aneurysm that had been surgically corrected two years before. On physical examination, several skin lesions were present: hypomelanotic macules, angiofibromas and hyperpigmented nevus on the face (Figure 1), neck, legs and arms. According to the patient, these lesions had been present since childhood. The neurological examination was unremarkable. Laboratory tests were normal. A brain MRI revealed the presence of cortical and subcortical tubers, a number of small calcified subependymal nodules in the walls of the lateral ventricles, and a nodular lesion with intense gadolinium enhancement located on the left lateral ventricle adjacent to the foramen of Monro, suggestive of a subependymal giant cell astrocytoma (Figure 2). A renal ultrasound showed multiple hyperechoic nodular formations and a subsequent abdominal MRI identified multiple AMLs and cystic for- 
Figure 1. Hyperpigmented nevus on the neck.

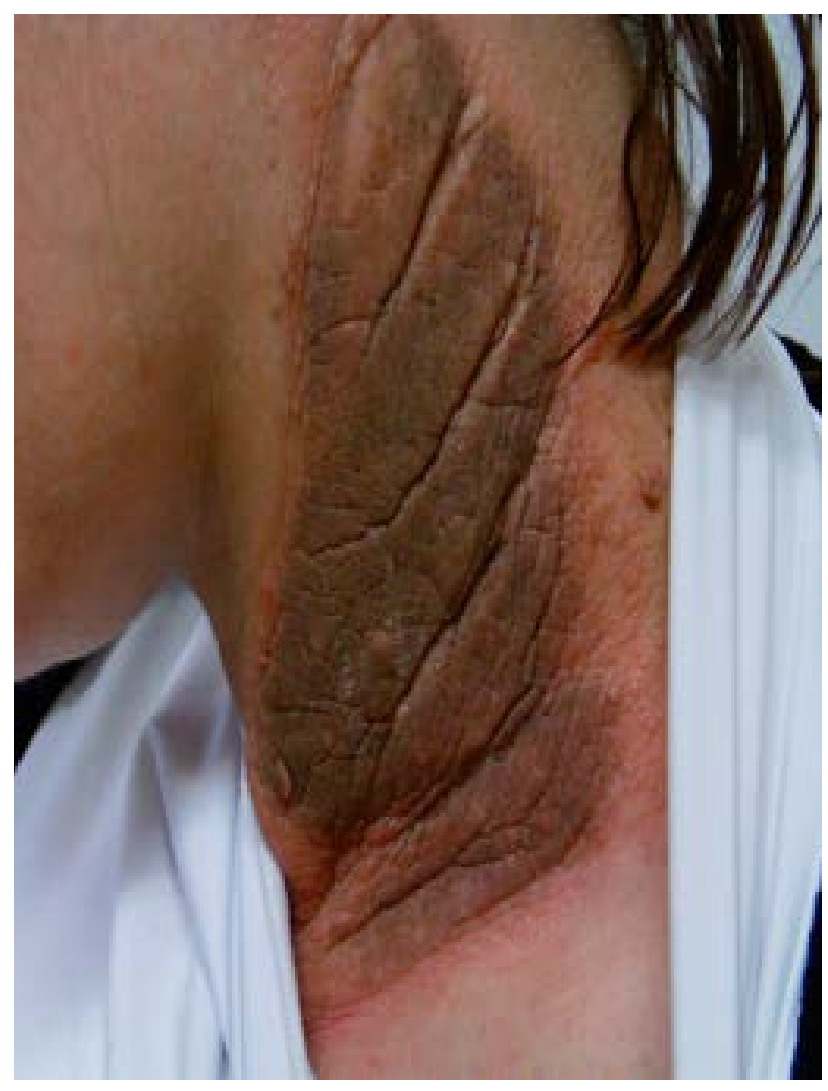

Figure 2. Subependymal giant cell astrocytoma - gadoliniumenhanced T1-weighted magnetic resonance imaging of the brain.

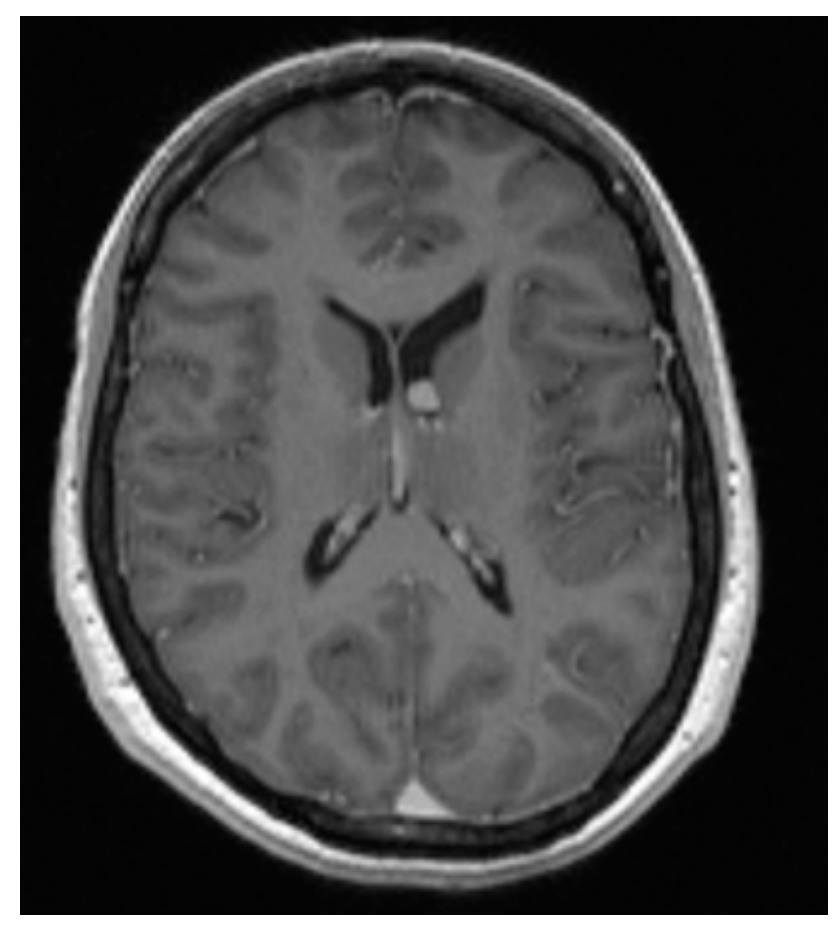

mations in both kidneys, as well as a liver haemangioma. A chest computed tomography (CT) did not show significant changes in lung interstitium, except for ground-glass opacities in the middle lobe adjacent to the large fissure. Echocardiography did not show any characteristic rhabdomyomas. An ophthalmological evaluation excluded retinal lesions and a dentistry workup revealed multiple gingival fibromas and a tongue lesion suggestive of an intraoral fibroma.

\section{Discussion}

Tuberous sclerosis complex is a rare entity that has a wide clinical spectrum of manifestations, with many patients having minimal signs and symptoms and no neurologic disability ${ }^{5,6}$. In fact, the classical Vogt's triad of adenoma sebaceum, seizures and mental retardation, described by Heinrich Vogt in 1908, is seen in less than half of the patients ${ }^{5,8,9}$. The variability of the clinical manifestations of TSC, including asymptomatic or minimally symptomatic adults, reinforces the importance of a detailed physical examination combined with several imaging modalities in achieving a correct diagnosis ${ }^{6}$. Diagnostic criteria for TSC have recently been updated by the Tuberous Sclerosis Complex Consensus Group ${ }^{10}$ - Table 1. Seizures are the most common neurological symptom, occurring in about $75 \%$ to $90 \%$ of individuals with tuberous sclerosis ${ }^{1,2}$. TSC is also associated with a wide variety of cognitive, behavioural and psychiatric manifestations. Brain MRI plays an important role in the diagnosis of TSC, allowing the identification of typical cerebral lesions such as tubers, subependymal nodules or subependymal giant cell astrocytomas, which may be present even in asymptomatic patients $2,3,5,9$. Skin lesions are also a common feature of TSC, being present in more than $90 \%$ of patients; these are generally detected during childhood or adolescence, although lesions can also appear in adulthood ${ }^{6}$. The most well-known cutaneous manifestation of TSC is facial angiofibroma (1). Renal manifestations of TSC include angiomyolipomas, isolated renal cysts, and renal cell carcinoma ${ }^{1,8}$. Renal disease is an important source of morbidity and mortality in patients with TSC, highlighting the importance of a continuous follow-up. Three forms of asymptomatic pulmonary involvement have been described: multifocal micronodular pneumocyte hyperplasia, pulmonary cysts and lymphangioleiomyomatosis. Recent prospective and retrospective studies have found cystic pulmonary abnormalities in as many as $40 \%$ of women with TSC $^{1,6}$. Other commonly recognized clinical features of TSC include retinal hamartomas, which are present in about 50\% of patients of any age, intraoral fibromas and dental enamel pits $^{11}$. On the contrary, vascular involvement is rare in tuberous sclerosis, particularly in adults.

The case described in this manuscript highlights the importance of recognizing the full spectrum of manifestations of TSC, including the most unusual, so not to miss the diagnosis and to allow correct treatment and improvement in quality of life ${ }^{9,10}$. Despite fulfilling clinical diagnostic criteria for definite TSC (Table 1), our patient was only diagnosed in adulthood, following the diagnosis of her young daughter (with a much 
Table 1.

\section{Clinical diagnostic criteria for Tuberous sclerosis (10)}

\section{Major features}

Hypomelanotic macules ( $\geq 3$, at least $5 \mathrm{~mm}$ diameter)

Facial angiofibromas $(\geq 3)$ or fibrous cephalic plaque

Ungual fibromas $(\geq 2)$

Shagreen patch (connective tissue nevus)

Multiple retinal hamartomas

Cortical dysplasia a (supra)

Subependymal nodules

Subependymal giant cell astrocytoma

Cardiac rhabdomyomas, single or multiple

Lymphangioleiomyomatosis b (supra)

Angiomyolipomas b (supra)

Definite diagnosis: Two major features or one

major feature with $\geq 2$ minor features

Possible diagnosis: Either one major feature or $\geq 2$ minor features

${ }^{a}$ Includes tubers and cerebral white matter radial migration lines.

${ }^{\mathrm{b}} \mathrm{A}$ combination of the two major clinical features

(lymphangioleiomyomatosis and angiomyolipomas) without

other features does not meet criteria for a definite diagnosis.

more severe phenotype). The reason for the delayed diagnosis relies on the fact that the patient is minimally symptomatic - apart from the typical skin lesions and the self-reported mild learning disability as a child, the other major manifestation of the disease is atypical. In fact, the presence of significant and potentially life-threatening vascular involvement (i.e. aortic arch aneurysm) is rarely associated with tuberous sclerosis in adulthood ${ }^{12}$. Most cases are diagnosed either incidentally, after aneurysm rupture or after presenting with hypertension, abdominal distention and abdominal or back pain ${ }^{12}$. Therefore, the recognition of TS and early diagnosis of vascular involvement are essential to prevent morbidity and mortality ${ }^{12}$. Another important aspect that should be recognized is familiar heterogeneity, which is also exemplified in this case report.

Management of tuberous sclerosis is symptomatic ${ }^{4}$, but an earlier diagnosis may allow a precocious surgical removal of the tumours, minimizing surgery-related morbidity and mortality ${ }^{1}$. Although there are no specific guidelines for the surveillance of this disease, continued vigilance and periodic imaging of the brain and abdomen is recommended to monitor the growth of lesions 6 .

\section{References}

1. Curatolo P, Maria BL. Tuberous Sclerosis. Handb Clin Neurol. 2013; 111:323-31.

2. Saxena A, Sampson JR. Epilepsy in Tuberous Sclerosis: Phenotypes, Mechanisms, and Treatments. Semin Neurol. 2015; 35:269-76.

3. Curatolo P, Moavero R, Vries PJ. Neurological and neuropsychiatric aspects of tuberous sclerosis complex. Lancet Neurol. 2015; 14: 733-45.

4. Curatolo P, Bombardieri R, Jozwiak S. Tuberous sclerosis. Lancet 2008; 372:657-68.

5. Sehgal VN, Singh N, Sharma S, Rohatgi J, Oberai R, Chatterjee K. Tuberous Sclerosis Complex: Diagnostic Role of Magnetic Resonance Imaging. Indian J Dermatol. 2015; 60:421.

6. Crino PB, Nathanson KL, Henske EP. The Tuberous Sclerosis Complex. N Engl J Med. 2006; 355:1345-56

7. von Ranke FM, Zanetti G, Pereira e Silva JL, Neto CAA, Godoy MCB, Souza CA, Manc AD, Souza Jr. AS, Escuissato DL, Hochhegger B, Maechiori E. Tuberous Sclerosis Complex: State-of-the-Art Review with a Focus on Pulmonary Involvement. Lung. 2015; 193:619-27.

8. Chawla A, Bosco J, Shenoy JN, Chinchure D. Giant renal angiomyolipomas in a patient with tuberous sclerosis. Indian J Nephrol. 2015; 25:257-8.

9. Umeoka S, Koyama T, Miki Y, Akai M, Tsutsui K, Togashi K. Pictorial review of tuberous sclerosis in various organs. Radiographics 2008; 28:e32.

10. Northrup H, Krueger DA, and on behalf of the International Tuberous Sclerosis Complex Consensus Group. Tuberous Sclerosis Complex Diagnostic Criteria Update: Recommendations of the 2012 International Tuberous Sclerosis Complex Consensus Conference. Pediatr Neurol. 2013; 49: 243-254.

11. Falsafi P, Taghavi-Zenouz A, Khorshidi-Khiyavi R, Nezami N, Estiar MA. A case of tuberous sclerosis without multiorgan involvement. Glob J Health Sci. 2015; 7:124-31.

12. Salerno AE, Marsenic 0, Meyers KEC, Kaplan BS, Hellinger JC. Vascular involvement in tuberous sclerosis. Pediatr Nephrol. 2010.; 25:1555-1561. 